\title{
Pruebas de fluidez verbal categoriales, fonológicas y gramaticales en la Infancia: Factores ejecutivos y semánticos
}

\author{
Categorical, phonological and grammatical verbal fluency tasks in Children: \\ Executive and semantic factors
}

Julián Marino ${ }^{1 *}$, Hugo Díaz-Fajreldines ${ }^{2,3}$

1 Facultad de Psicología. Universidad Nacional de Córdoba. Córdoba, Argentina.

2 Instituto Privado de Neurociencias, Córdoba. Córdoba, Argentina.

3 Facultad de Ciencias Químicas. Universidad Nacional de Córdoba. Córdoba, Argentina.

\section{Resumen}

En el presente trabajo se aplicaron las Pruebas de Fluidez Verbal (PFV), correspondientes al Explorador Neuropsicológico en Fluidez Verbal (ENFV), a una población de niños argentinos. El objetivo principal fue conocer su estructura factorial en niños y divulgar valores normativos. Se obtuvieron dos factores principales: uno ejecutivo y el otro semántico. Esto avala gran parte de la literatura especializada, que discute sobre el rol de las capacidades atencionales y otras habilidades en PFV. Respecto del rol de la edad, se encontró una elevada incidencia en todas las pruebas, excepto la categorial de supermercado. La influencia fue mayor para las pruebas fonológicas y de verbos, lo que fue relacionado con su mayor compromiso frontal y la tardía maduración de estas estructuras. Se sugiere, por último, la continuidad de estos trabajos desde una perspectiva interactiva entre nodos ejecutivos y semánticos. Esto conlleva la utilización de equipos de neuroimágenes para obtener imágenes por Tensor de Difusiones, cuyo dato principal es la conectividad entre regiones cerebrales distantes.

Palabras clave: fluidez verbal, funcionamiento ejecutivo, procesamiento semántico, estructura factorial, control ejecutivo semántico

\section{Abstract}

In this work we applied Verbal Fluency Tasks (VFT) belonging to the Verbal Fluency Neuropsychological Explorer (VFNE) to a population of Argentinean childrens. The main objective was to determine the factor structure in children and disseminate normative values. We obtained two main factors: one executive and one semantic. This supports much of the literature, which discusses the role of attentional capacities and other skills in VFT. On the role of Age, there was a high incidence in all tests except the categorical supermarket. The influence was greater for the phonological tests and verbs, which was associated with greater commitment and delayed frontal maturation of these structures. It suggests, finally, the continuity of this work from an interactive perspective between executive and semantic nodes. This involves the use of neuroimaging equipment to obtain diffusion tensor images, which shows connectivity data between distant brain regions.

Keywords: verbal fluency, executive functioning, semantic processing, semantic executive control system

\footnotetext{
* Correspondencia: jmarino@psyche.unc.edu.ar. Facultad de Psicología, Universidad Nacional de Córdoba. 25 de Mayo 912, 1B. Córdoba, X5000, Argentina

Recibido: 25-03-2011. Revisión desde: 06-04-11. Aceptado: 10-06-2011

DOI: $10.5839 /$ rcnp.2011.0601.08
} 


\section{Introducción}

Las Pruebas de Fluidez Verbal (PFV) son técnicas neuropsicológicas que implican la actividad de diversos procesos cognitivos, tales como operaciones ejecutivas, mecanismos de control, atencionales y memoria semántica (Gauthier, Duyme, Zanca \& Capron, 2009; Henry \& Crawford, 2004; Koren, Kofman \& Berger, 2005). Estas pruebas consisten en la evocación de un tipo determinado de palabras en una cantidad de tiempo específica. El tipo de palabras a evocar varía sistemáticamente de acuerdo con categorías estandarizadas, como las semánticas, fonológicas, gramaticales, duales (Marino \& Alderete, 2010; Schwartz \& Baldo, 2001; Schwartz, Baldo, Graves \& Brugger, 2003) y combinaciones entre las anteriores (Marino \& Alderete, 2010).

Estas variaciones son importantes para determinar la activación cognitiva y cerebral específica de la fluidez verbal, existiendo una amplia cantidad de trabajos comparativos acerca de cómo los cambios en tipos de PFV provocan un involucramiento diferencial en los procesos y estructuras cognitivas (Brickman et al., 2005). También, existe una vasta literatura en neuroimágenes y lesiones cerebrales que relaciona estos cambios con patrones de activación cerebral específicos (Baldo \& Shimamura, 1998; Bozikas, Kosmidis \& Karavatos, 2005; Vitali et al., 2005). Por ejemplo, se ha encontrado que la PFV de acción (nombramiento de verbos) implica una actividad focalizada en la corteza motora primaria y secundaria, así como en el giro frontal inferior izquierdo y los ganglios de la base y cuerpo estriado (Ostberg et al., 2007; Piatt, Fields, Paolo \& Tröster, 1999). Esto le asigna una utilidad clínica particular cuando se trata de evaluar posibles demencias por enfermedad de Parkinson y HIV (Fernaeus, Ostberg, Almkvist \& Wahlund, 2000; Ostberg et al., 2007; Piatt et al., 1999; Tröster, Woods, Fields, Hanisch \& Beatty, 2002). En cambio, en PFV semánticas, como el nombramiento de animales, se halló un patrón de activación cerebral extendido hacia porciones temporales y occipitales de la corteza cerebral (Damasio, Tranel, Grabowski, Adolphs \& Damasio, 2004), manteniéndose la activación del giro frontal inferior izquierdo (Vitali et al., 2005).

Sin embargo, dentro de las PFV semánticas se encontraron diferencias por tipo de concepto, por ejemplo, en evocación de Frutas hay una mayor activación occipital que en Animales, y en evocación de Herramientas hay un involucramiento parietal superior y frontal motor claramente diferenciado de las PFV anteriores (Damasio et al., 2004; Pulvermüller, 2002). Esto indica que, aun dentro de un mismo tipo de PFV, hay propiedades y dimensiones subyacentes que provocan diferencias en la actividad nerviosa. Una de las explicaciones vertidas sobre estos hallazgos consiste en que la evocación de conceptos adquiere su patrón de activación cerebral de acuerdo con la proporción que tengan de rasgos (visuales, de movimiento, sensoriales) (Pulvermüller, 2002). Así, podría explicarse por qué una categoría como Animales, con fuertes rasgos visuales, de movimiento y sonido, tiene mayor compromiso temporal que la categoría Frutas, de mayor relación con rasgos visuales y olfativos. Estas variaciones intra- tipo también se encontraron en las PFV fonológicas, respecto de dimensiones como la disponibilidad léxica del universo de palabras y el carácter directo o indirecto de la consigna (Bryan, Luszcz \& Crawford, 1997; Dräger et al., 2004; Fu et al., 2006). Por ejemplo, Dräger et al. (2004) pidieron la evocación de palabras en un sentido progresivamente restrictivo respecto del universo total de palabras posibles, siguiendo criterios exclusivamente fonológicos, y hallaron un mayor compromiso de la actividad frontal en las pruebas de menor disponibilidad léxica en comparación con las de mayor disponibilidad. En cuanto a tipos de consignas, se encontró una mayor actividad de la memoria de trabajo cuando la consigna implicó decir palabras que no contuvieran determinada letra (consigna indirecta), en comparación con las mencionadas de letra inicial (consigna directa) (Troyer, Moscovitch, Winocur, Alexander \& Stuss, 1998). Sin embargo, más allá de estas distinciones, tanto dentro como fuera de un tipo de PFV, en la literatura la clasificación más frecuentemente utilizada implica, por un lado, las de compromiso mayormente frontal (fonológicas y verbos) y, por otro, las que tienen una mayor actividad temporo- parietal (semánticas).

Teniendo en cuenta lo anterior, el objetivo del presente estudio fue aplicar una batería completa de PFV, incluyendo semánticas, fonológicas, gramaticales y combinadas, en una población infantil, para conocer el comportamiento de una gran variedad de PFV, aplicadas en conjunto, y determinar si los factores latentes detrás de las mismas respondían a las distinciones conceptuales intra y entre los tipos de PFV, o bien seguían una organización más simple, de tipo dual (Henry \& Crawford, 2004). Esto es importante de resolver en función del objetivo mayor de una serie de investigaciones, que consiste en diseñar una batería de PFV, lo más sistemática y amplia posible, para explorar diferentes funciones cognitivas mediante el conocimiento de la organización que preside la evocación de palabras (Explorador Neuropsicológico en Fluidez Verbal) (ENFV) (Marino \& Alderete, 2010). El ENFV es un pool de PFV asociadas a diferentes actividades cerebrales, conformando una batería breve que intenta aprovechar la sencillez y versatilidad de estas pruebas, y que resulta utilizable en contextos clínicos en los que la evaluación neuropsicológica es dificultosa, como las internaciones hospitalarias.

Aún no resulta claro si las diferencias observadas mediante las técnicas de neuroimagen y las pruebas cognitivas se reflejan en la estructura subyacente de los puntajes obtenidos en las PFV. El Análisis Factorial es una herramienta sumamente útil para indagar si la estructura del rendimiento en PFV, medida por el criterio cuantitativo, responde a las clasificaciones teóricamente esperables. En otras palabras, si un conjunto de PFV semánticas, fonológicas, gramaticales y combinadas suponen factores del mismo tipo. Este es un asunto de importancia, porque en las PFV se desarrollan procesos cognitivos - como la búsqueda de palabras, la generación de estrategias, el monitoreo del procesamiento y el almacén semántico- que trascienden el tipo de prueba utilizada. Entonces, ¿̇en qué medida estos procesos comunes se relacionan con las diferencias particulares de cada PFV como para explicar el rendimiento cuantitativo de las personas mediante factores diferentes? La clasificación de PFV en semántica, fonológica, gramatical y combinada, ¿se ve reflejada en la estructura factorial de un conjunto de PFV, o hay factores comunes que trascienden la clasificación tradicional, por otras dimensiones que deberían ser estudiadas?

El interés también reside en conocer la estructura factorial de las PFV en población infantil, siendo que en esta etapa evolutiva hay importantes cuestiones de desarrollo neuronal que impactan en cada uno de los procesos cognitivos vinculados con la recuperación de palabras (Best, Miller \& Jones, 2009). Por lo tanto, puede haber diferencias en la organización de la fluidez verbal en esta etapa respecto de la adultez, ya que las 
estructuras cerebrales vinculadas primordialmente con las funciones ejecutivas están en pleno proceso de maduración (Huettel, Misiurek, Jurkowski \& McCarthy, 2004; Huizinga, Dolan \& Van der Molen, 2006; Miyake et al., 2000). Se ha observado que el control inhibitorio, la memoria de trabajo y la flexibilidad cognitiva se encuentran entre los 8 y 14 años en un nivel intermedio respecto del nivel adulto (Johnson, 2003; Miyake et al., 2000).

A la vez, de una importancia teórica crucial, se considera que los factores que es necesario extraer deberían estar correlacionados entre sí, cuestión que obedece a la conocida "impureza" de los procesos cognitivos (Miyake et al., 2000), y superpuestos unos con otros, tanto en su intercambio informacional como en su arquitectura nerviosa. Por ejemplo, se ha demostrado, mediante estudios funcionales y tractografías, que no es posible conceptualizar una operación ejecutiva de forma absolutamente independiente de procesos semánticos o procesos atencionales (De Zubicaray, Rose \& McMahon, 2010; Voineskos et al., 2010). Cuando se estimula un proceso cognitivo mediante una tarea, se advierten activaciones en red que revelan compromisos distribuidos de capacidades varias. Consecuentemente, los factores latentes a extraer de una matriz de PFV deben contemplar una posible correlación entre sí, siendo entonces una rotación oblicua de los factores el método adecuado. Este tipo de rotación, según diversos autores, suele alcanzar mayor relevancia y representatividad teórica que las rotaciones ortogonales (Hair, Anderson, Tatham \& Black, 1999). A la vez, en esta investigación se presentan valores normativos para niños de 8 a 14 años de edad, en ambos sexos, incluyéndose las ecuaciones de regresión sobre cada PFV, ingresando como predictores el sexo y la edad.

\section{Construcciones hipotéticas}

Se espera hallar una estructura factorial que, ante la variedad de PFV, represente las distinciones mayores: Fonológicas, Semánticas y Gramaticales, cada una significando un factor. El papel de las PFV combinadas tiene mayor nivel exploratorio, ya que son relativas a cada estudio, siendo muy particulares las combinaciones que concretamente se efectúan. Por ejemplo, en un estudio anterior se combinaron nombres propios geográficos con letras finales; en el presente estudio se utilizan nombres propios de personas también combinados con letra final. En este sentido, puede haber una dimensión de consigna indirecta que acerque estas pruebas a la PFV de consigna indirecta, de tipo fonológica.

Dado que todas las pruebas aplicadas son genéricamente PFV, se espera que un alto porcentaje de varianza resulte explicado por los factores extraídos. Por esta misma razón, se espera que los valores de esfericidad resulten apropiados para emprender este tipo de análisis de datos.

\section{Materiales y métodos}

\section{Población}

Participaron del estudio 156 niños (78 varones) de escuelas primarias y secundarias de la ciudad de Córdoba (Argentina). Los establecimientos fueron de carácter público y semipúblico, correspondiendo a un extracto social de clase media. La distribución por edad fue la siguiente: 8 años $(N=19)$; 9 años $(N=22)$;
10 años $(N=36) ; 11$ años $(N=15) ; 12$ años $(N=21) ; 13$ años $(N=$ 21) y 14 años $(N=22)$. Los niños incluidos en el análisis no tenían alteraciones neurológicas ni problemas cognitivos diagnosticados. En los casos en que las autoridades de los establecimientos educativos revelaron la existencia de problemas de aprendizaje, las pruebas igualmente fueron realizadas, pero no incluidas en el análisis ( $N=21)$.

\section{Procedimiento}

Las evaluaciones se realizaron en las escuelas, durante el horario de clase matutino. Las sesiones consistieron en la aplicación del set de PFV y una batería de pruebas neuropsicológicas de Funciones Ejecutivas. Las PFV se aplicaron en primer término, siguiendo siempre el mismo orden: Animales, Frutas, Supermercado, Cuerpo, Letra $P$, Letra $F$, Letra Excluida A, Verbos, Adjetivo, Nombres de Mujer, Nombres de Varón. Las evocaciones se grabaron mediante un grabador digital de voces Panasonic RR- US511. Fueron sesiones individuales, conducidas por el primer autor de este trabajo y dos tesistas de Licenciatura en Psicología, especialmente capacitadas para la evaluación neuropsicológica. Cada sesión duró aproximadamente una hora.

\section{Instrumentos}

Consigna: Las 11 PFV duraron un minuto cada una y en todos los casos se alentó a los niños a decir la mayor cantidad de palabras posible en ese tiempo. Para el análisis de esta investigación se computó la cantidad de palabras correctas evocadas en cada prueba.

Las PFV aplicadas fueron:

a) Semánticas:

a1 Animales: Se debía evocar la mayor cantidad posible de animales. En el análisis no se computaron las categorías supraordinales ("mamíferos", "peces") ni las subordinadas (razas de perros). Tampoco las repeticiones, lo cual se mantuvo para todas las pruebas.

a2 Frutas: Se debía evocar la mayor cantidad posible de frutas.

a3 Partes del Cuerpo Humano: Se le pidió a los niños que nombraran la mayor cantidad posible de partes del cuerpo humano. Los niveles de organización de las partes evocadas se dejaron a criterio del niño (por ejemplo, hubo niños que evocaron partes de una célula, considerándose válidas las respuestas).

a4 Supermercado: Se solicitó que evocaran todas las cosas que pueden comprarse en un supermercado. No se computaron las marcas. En esta prueba, sobre todo en los niños más pequeños, hubo varias "superposiciones categoriales", como decir electrodomésticos y luego licuadora. Se computaron como válidas las dos respuestas. Más allá de la puntuación cuantitativa, esta prueba se recomienda para evaluar la maduración de la organización categorial de los niños.

\section{b) Fonológicas:}

b1 Letra inicial P: Se pidió que dijeran la mayor cantidad de palabras que empiecen con la letra $\mathrm{P}$. Se aclaró que no podían ser familias de palabras (por ejemplo, no era válido decir "perro", "perra", "perrito") ni nombres propios.

b2 Letra inicial F: Se mantuvieron los criterios de la Letra inicial P. 
b3 Letra excluida A: Se pidió que evocaran la mayor cantidad posible de palabras sin la letra $A$ en ninguna de sus sílabas. Se mantuvieron los criterios aplicados en Letra P y Letra F.

c) Gramaticales:

c1 Verbos: Se pidió que evocaran la mayor cantidad posible de verbos terminados en AR, ER o IR (infinitivo).

c2 Adjetivos Calificativos Positivos: Se pidió que evocaran la mayor cantidad posible de adjetivos calificativos que se puedan dirigir a una persona, y que tengan una connotación positiva. Se brindó como ejemplo: "Lindo" y "Bueno".

d) Combinadas:

d1 Nombre de Mujer terminado en la letra A: Se pidió que evocaran la mayor cantidad posible de nombres de mujer terminados en letra $A$.

d2 Nombres de Varón terminados en la letra O: Se pidió que evocaran la mayor cantidad posible de nombres de varón terminados en $\mathrm{O}$. Se repasó el concepto de consonante antes de empezar la prueba.

\section{Resultados}

Descriptivos y análisis de normalidad de las variables.

Como se señaló en el apartado "Instrumentos", el dato utilizado por PFV fue la cantidad de palabras correctas por prueba. A continuación se presenta la media y desviación estándar para cada PFV, así como sus índices de curtosis y asimetría.

Considerando que para el desarrollo del ENFV cada PFV actúa como un ítem del mismo, se calculó un índice de fiabilidad: el Alfa de Cronbach para el total de las pruebas fue de 0.843 (11 elementos), considerándose muy adecuado.

Ecuaciones de Regresión: Edad y Sexo por PFV.

Como uno de los objetivos de este estudio es brindar valores normativos para una población argentina en estas pruebas, se presentan las ecuaciones de regresión sobre cada PFV ingresando Edad y Sexo como predictoras. En el caso del Sexo, se creó una variable ficticia, siendo $1=$ varón y 2 = mujer. La regresión se realizó mediante un método jerárquico, hacia adelante, con un valor de entrada de 0.05 y un valor de salida de 0.10 . Se encontró solo un modelo no significativo (PFV Supermercado). Se presentan los valores de B no estandarizados para que puedan calcularse los valores normativos (ver tabla 2).

\section{Medidas de adecuación para realizar un Análisis Factorial.}

La medida de adecuación muestral de Kayser-Meyer-Olkin arrojó un valor de 0.863 , lo que indica una alta proporción de varianza común. Este estadístico apoya la utilidad de realizar un Análisis Factorial con estos datos. Por su parte, la Prueba de Esfericidad de Bartlett tuvo una significación de 0.000 (chi $^{2}=$ 544.06), sumándose al estadístico anterior sobre la adecuación de la matriz de correlaciones. El examen de las matrices antiimagen permitió observar en la diagonal valores de adecuación a la muestra (VAM) superiores a 0.80 , en todos los casos. De este modo, los estudios previos, sumados a los de normalidad, revelan que es posible el Análisis Factorial.

\section{Análisis de residuos.}

Mediante la matriz de correlaciones reproducidas se compararon las correlaciones observadas y las reproducidas. Esto arrojó 29 residuales no redundantes, con valores superiores a 0.05 . Esto implica el $52 \%$ del total de las correlaciones, lo que indica que el modelo obtenido tiene un ajuste moderado.

\section{Autovalores.}

Se procedió a la extracción de factores mediante el método de componentes principales con rotación oblicua oblimin-max (Hair, et al., 1999). Se utilizó como punto de corte autovalores superiores a 1 (ver tabla 3 y Screen Plot gráfico 1).

Al efectuarse una rotación oblicua, se obtuvieron las matrices de configuración y de estructuras (ver tablas 5 y 6 ). La correlación obtenida entre los factores fue de 0.52 , lo que justifica ampliamente la utilización de una rotación oblicua.

\section{Análisis con tres factores a priori.}

Teniendo en cuenta que se encontró un tercer factor que alcanza un autovalor de 0.982 , muy cercano al punto de corte, se procedió a realizar un análisis factorial con número preestablecido de factores (3), para conocer de forma exploratoria cuál es la estructura del tercero.

Se obtuvo una varianza explicada de $60.86 \%$ y se observó que en las nuevas matrices de configuración y de estructura se mantuvo la pertenencia de las variables a los factores, al igual que en el análisis principal de esta investigación. Sin embargo, una de las PFV, Adjetivos Calificativos, pasó a liderar la saturación del tercer factor, con una correlación de 0.711 en la matriz de configuración y 0.739 en la matriz de estructura, en oposición a las cargas de 0.125 (factor 1) y 0.302 (factor 2) en la matriz de configuración. Esto indica que la tracción hacia el tercer factor se encontraba forzada por la PFV Adjetivos Calificativos.

\begin{tabular}{lcccc}
\hline & $\mathrm{M}$ & $\mathrm{Ds}$ & Asimetría & Curtosis \\
\hline Animales & 15,78 & 4,436 &, 113 &,- 210 \\
Frutas & 9,66 & 2,531 &,- 056 &,- 543 \\
Cuerpo & 16,09 & 5,367 &, 089 &,- 226 \\
Supermercado & 14,04 & 5,028 &, 394 &, 060 \\
Letra P & 9,28 & 3,708 &, 351 &, 326 \\
Letra F & 6,15 & 3,004 &, 559 &, 041 \\
Letra Excl. A & 5,88 & 2,573 &, 194 &,- 221 \\
Verbos & 11,04 & 4,769 &, 617 &, 365 \\
Adjetivos & 6,90 & 3,175 &, 923 &, 804 \\
Nombres Mujer & 11,00 & 4,086 &, 635 &, 593 \\
Nombres Varón & 5,77 & 2,747 &, 210 &,- 272 \\
\hline
\end{tabular}

Tabla 1. Estadísticos descriptivos y valores de Asimetría y Curtosis para cada PFV. 
Marino, et al. Rev. Chil. Neuropsicol. 6(1): 47-54, 2011

\begin{tabular}{lccccccc}
\hline & $\mathrm{R}^{2}$ corregida & constante & $\mathrm{b}^{*}$ Edad $^{\mathrm{a}}$ & $\mathrm{b}^{*}$ Sexo & Error & $\mathrm{F}$ & Sig. \\
& & & & & & & \\
\hline Animales & 0.131 & 11.74 & 0.636 & -1.954 & 4.13 & 12.64 & $0.000^{* *}$ \\
Frutas & 0.077 & 5.60 & 0.370 & No sig. & 2.43 & 13.85 & $0.000^{* *}$ \\
Cuerpo & 0.081 & 7.77 & 0.759 & No sig. & 5.17 & 12.87 & $0.000^{* *}$ \\
Supermercado & & & El modelo resultó no significativo. & & \\
Letra P & 0.137 & 1.582 & 0.703 & No sig. & 3.44 & 24.78 & $0.000^{* *}$ \\
Letra F & 0.140 & -0.226 & 0.583 & No sig. & 2.79 & 26.14 & $0.000^{* *}$ \\
Letra Excl. A & 0.121 & 0.772 & 0.467 & No sig. & 2.41 & 22.41 & $0.000^{* *}$ \\
Verbos & 0.123 & 1.493 & 0.872 & No sig. & 4.46 & 22.81 & $0.000^{* *}$ \\
Adjetivos & 0.087 & 0.402 & 0.397 & 1.430 & 3.10 & 8.41 & $0.000^{* *}$ \\
Nombres Mujer & 0.286 & -3.756 & 0.997 & 2.563 & 3.67 & 31.97 & $0.000^{* *}$ \\
Nombres Varón & 0.232 & -1.690 & 0.681 & No sig. & 2.40 & 47.87 & $0.000^{* *}$ \\
\hline
\end{tabular}

Tabla 2. Ecuaciones de regresión sobre cada PFV. Edad y Sexo ingresan como variables predictoras.

${ }^{a}$ Los valores de b son no estandarizados, ${ }^{b}$ Sexo es variable ficticia con valores 2 = mujer y $1=$ varón, ${ }^{*}=$ significativo a nivel 0.05 ,

$* *$ s significativo a nivel 0.01

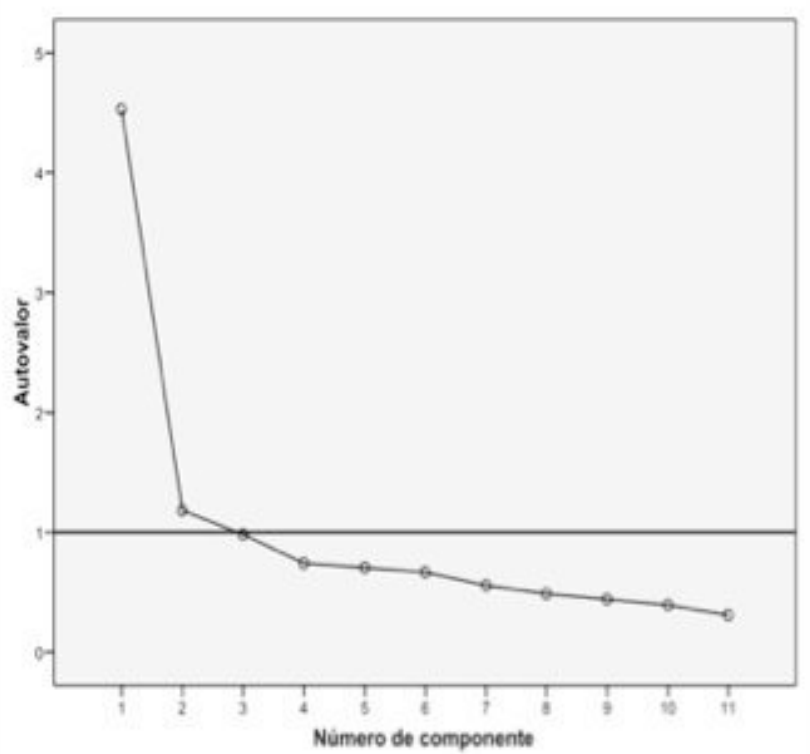

Gráfico 1. Sedimentación de los factores hallados.

Autovalores en el eje de las ordenadas y número de factor en las abscisas. El autovalor 1 es el punto de corte establecido para la extracción.

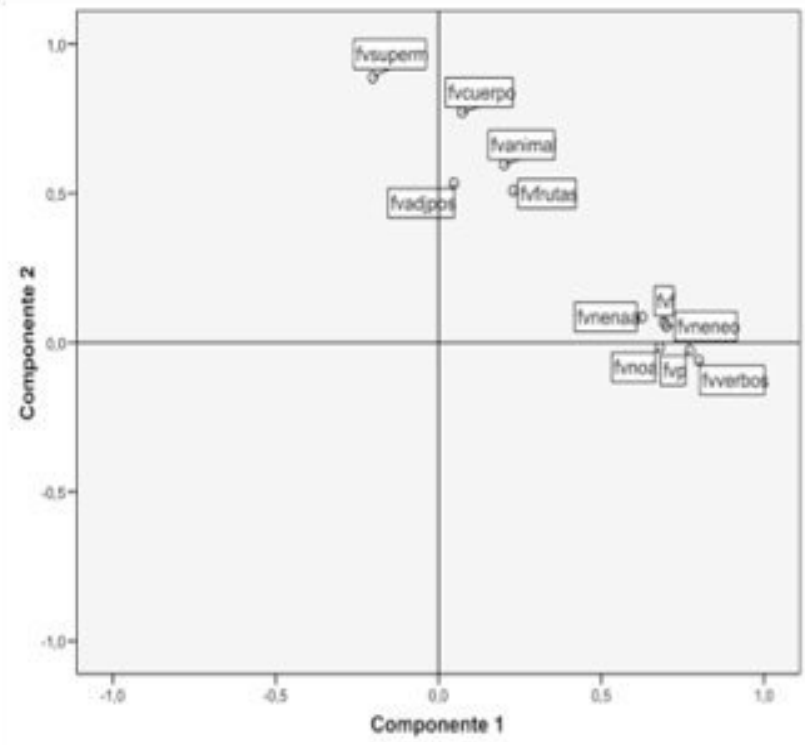

Gráfico 2. Componentes en espacio rotado. 


\begin{tabular}{cccc}
\hline Factores & Autovalores & $\begin{array}{c}\text { Proporción de la } \\
\text { varianza explicada }\end{array}$ & $\begin{array}{c}\text { Porcentaje acumu- } \\
\text { lado explicado }\end{array}$ \\
\hline $\mathbf{1}$ & $\mathbf{4 , 5 3 0 *}$ & $\mathbf{4 1 , 1 8 2}$ & $\mathbf{4 1 , 1 8 2}$ \\
$\mathbf{2}$ & $\mathbf{1 , 1 8 4 *}$ & $\mathbf{1 0 , 7 6 1}$ & $\mathbf{5 1 , 9 4 3}$ \\
3 &, 982 & 8,925 & 60,868 \\
4 &, 742 & 6,749 & 67,617 \\
5 &, 705 & 6,409 & 74,026 \\
6 &, 667 & 6,062 & 80,087 \\
7 &, 557 & 5,061 & 85,148 \\
8 &, 488 & 4,434 & 89,583 \\
9 &, 442 & 4,018 & 93,601 \\
10 &, 394 & 3,586 & 97,187 \\
11 &, 309 & 2,813 & 100,000 \\
\hline
\end{tabular}

Tabla 3. Autovalores de los factores extraídos y proporción de la varianza explicada (Método de extracción: Componentes principales).

\begin{tabular}{|c|c|c|}
\hline & Factor 1 & Factor 2 \\
\hline Animales & 202 & ,597 \\
\hline Frutas & 230 & ,509 \\
\hline Cuerpo & ,073 & ,772 \\
\hline Supermercado &,- 202 & ,889 \\
\hline Letra P & ,773 &,- 027 \\
\hline Letra F & 693 & ,067 \\
\hline Letra Excl. A & 679 &,- 017 \\
\hline Verbos & ,800 &,- 060 \\
\hline Adjetivos & ,048 & ,534 \\
\hline Nombres Mujer & ,626 & ,085 \\
\hline Nombres Varón & 701 & ,054 \\
\hline
\end{tabular}

Tabla 5. Matriz de Configuración: Saturación de los factores extraídos en cada variable.

Método de extracción: Análisis de componentes principales. Método de rotación: Normalización Oblimin con Kaiser. La correlación entre los dos factores fue de 0.52 .

\begin{tabular}{lc}
\hline PFV & Comunalidades \\
\hline Animales &, 522 \\
Frutas &, 433 \\
Cuerpo &, 660 \\
Supermercado &, 645 \\
Letra P &, 576 \\
Letra F &, 533 \\
Letra Excl. A &, 450 \\
Verbos &, 593 \\
Adjetivos &, 314 \\
Nombres Mujer &, 454 \\
Nombres Varón &, 533 \\
\hline
\end{tabular}

Tabla 4 . Aporte de comunalidades por PFV. Método de extracción: Análisis de Componentes principales.

\begin{tabular}{lcc}
\hline & Factor 1 & Factor 2 \\
\hline Animales &, 512 & $\mathbf{, 7 0 2}$ \\
Frutas &, 494 & $\mathbf{6 2 8}$ \\
Cuerpo &, 474 & $\mathbf{, 8 1 0}$ \\
Supermercado &, 260 & $\mathbf{, 7 8 5}$ \\
Letra P & $\mathbf{, 7 5 9}$ &, 375 \\
Letra F & $\mathbf{, 7 2 8}$ &, 427 \\
Letra Excl. A & $\mathbf{, 6 7 1}$ &, 336 \\
Verbos & $\mathbf{, 7 6 9}$ &, 356 \\
Adjetivos & $\mathbf{3 2 5}$ & $\mathbf{, 5 5 8}$ \\
Nombres Mujer & $\mathbf{6 7 0}$ &, 410 \\
Nombres Varón & $\mathbf{, 7 2 9}$ &, 418 \\
\hline
\end{tabular}

Tabla 6. Matriz de Estructura para la saturación de los factores extraídos sobre cada PFV teniendo en cuenta las correlaciones entre los factores.

Método de extracción: Análisis de componentes principales.

Método de rotación: Normalización Oblimin con Kaiser.

\section{Discusión}

Los resultados obtenidos indican que la varianza de PFV en niños resulta explicada por 2 factores en poco más de un $50 \%$. Esto coincide con parte de la literatura especializada, que propone la existencia principalmente de factores Ejecutivo y Semántico (Marino \& Alderete, 2010; Schwartz et al., 2003; Troyer, Moscovitch \& Winocur, 1997), y difiere con la inclusión de una mayor cantidad de factores, como capacidades atencionales y de velocidad (Henry \& Crawford, 2004). El Factor 1 se interpreta como ejecutivo debido a su correlación con las PFV verbos, fonológicas directas, fonológica indirecta y nombres propios (con restricciones fonológicas). En estudios con neuroimágenes, todas las pruebas mencionadas evidenciaron alto compromiso prefrontal (Dräger et al., 2004; Fu et al., 2000; Fu et al., 2006; Ostberg et al., 2007). Por su parte, el Factor 2 se considera de actividad semántica. Tuvo alta correlación para PFV categoriales, destacándose la de Supermercado.

Sin embargo, entre los dos factores se halló una elevada correlación. Esto justifica la elección de un método de rotación oblicuo y, teóricamente, refuerza la noción de que los procesos semánticos y ejecutivos son altamente interactivos en PFV. La base neuroanatómica de esta interacción son los fascículos fronto-temporales, cuya conectividad es tema de intenso estudio en la actualidad (Catani \& Mesulam, 2008; Hasan et al., 2009). Cognitivamente es la base del Control Semántico (Jefferies, Patterson \& Ralph, 2008; Noonan, Jefferies, Corbett \& Lambon Ralph, 2009).

El tercer factor extraído representó el comportamiento de la PFV de Adjetivos Calificativos dirigidos hacia una persona. Esta prueba difiere de todas las restantes, ya que implica la evocación de adjetivos con una restricción contextual específica. No existen antecedentes de estudios sobre esta prueba, por lo que resultan necesarias investigaciones sobre sus bases neuroanatómicas y aplicaciones clínicas. 
En cuanto a las propiedades que arrojó cada prueba, la mayor cantidad de palabras fue evocada en las categoriales. En comparación con población adulta (Marino \& Alderete, 2010), se observó que los niños alcanzaron un rendimiento muy cercano en pruebas categoriales y más lejano en las fonológicas. Esto podría estar relacionado con la maduración tardía de las estructuras frontales. La Edad (maduración intrainfancia) influyó fuertemente en casi el total de las pruebas, en especial en nombres propios y verbos. Estudios de neuroimágenes y neuropsicológicos reconocen que el almacenamiento de nombres propios es más tardío y débil que el de categorías y conceptos comunes, y depende de estructuras nerviosas más puntuales (Pulvermüller, 2002; Shtyrov, Kujala \& Pulvermüller, 2010). La Edad no tuvo influencia en la prueba de Supermercado.

La PFV Frutas evidenció una escasa dispersión de sus puntajes $y$, dado su elevado compromiso con el factor iniciación, se considera una prueba de carácter motivacional en la que los valores no difieren demasiado entre personas. Fue la prueba categorial con mayor correlación con el factor ejecutivo. Por su parte, la PFV Animales tuvo un alto compromiso con la variable Edad y con el Sexo, a favor de los varones. La PFV Supermercado, como se señaló, tuvo la carga más elevada en el factor Semántico y una correlación negativa con el factor Ejecutivo. Esto avala los antecedentes que utilizan esta prueba como medida de la capacidad de categorización, con complejas relaciones en niveles supraordinales, típicos y subordinados. En diferentes estudios se emplea PFV Supermercado de un modo cualitativo, como criterio de organización del espacio conceptual (Lezak, 1995). La PFV Partes del Cuerpo tuvo una elevada carga en el Factor Semántico y la media más alta de producción de todo el ENFV. Contiene, al igual que Supermercado, posibilidades de categorización compleja, pero más restringida que en aquella prueba. En cuanto a Verbos, su carga en el primer factor confirmó su carácter predominantemente frontal. Fue una de las pruebas que más aportó a la comunalidad. Estudios previos, clínicos y de neuroimagen revelaron su compromiso frontal-motor. Esto indica que, más allá del compromiso frontal compartido, existen diferencias especificas dentro del mismo.

Entre las pruebas de letra inicial no se hallaron diferencias notables: se encontró que la letra $\mathrm{P}$ tuvo mayor productividad que $F$, lo cual resulta esperable dada su mayor disponibilidad léxica. Por su parte, Letra Excluida tuvo menor productividad, influencia de la edad y aporte comunal.

En síntesis, el ENFV puede resultar un instrumento útil para evaluar el funcionamiento ejecutivo semántico en población infantil. Se requieren nuevos estudios sobre conectividad para abordar su desempeño desde una perspectiva de especificidad interactiva.

\section{Referencias}

Baldo, J.V. \& Shimamura, A.P. (1998). Letter and Category Fluency in Patients With Frontal Lobe Lesions. Neuropsychology, 12(2), 259267.

Best, J.R., Miller, P.H. \& Jones, L.L. (2009). Executive functions after age 5: Changes and correlates. Developmental Review, 29(3), 180-200.

Bozikas, V.P., Kosmidis, M.H. \& Karavatos, A. (2005). Disproportionate impairment in semantic verbal fluency in schizophrenia: differential deficit in clustering. Schizophrenia Research, 74(1), 51-59.
Brickman, A.M., Paul, R.H., Cohen, R.A., Williams, L.M., MacGregor, K.L., Jefferson, A.L., et al. (2005). Category and letter verbal fluency across the adult lifespan: relationship to EEG theta power. Archives of Clinical Neuropsychology, 20(5), 561-573.

Bryan, J., Luszcz, M.A. \& Crawford, J.R. (1997). Verbal Knowledge and Speed of Information Processing as Mediators of Age Differences in Verbal Fluency Performance Among Older Adults. Psychology and Aging, 12(3), 473-478.

Catani, M. \& Mesulam, M. (2008). The arcuate fasciculus and the disconnection theme in language and aphasia: History and current state. Cortex, 44(8), 953-961.

Damasio, H., Tranel, D., Grabowski, T., Adolphs, R. \& Damasio, A. (2004). Neural systems behind word and concept retrieval. Cognition, 92(1-2), 179-229.

De Zubicaray, G.I., Rose, S.E. \& McMahon, K.L. (2010). The structure and connectivity of semantic memory in the healthy older adult brain. Neuroimage, In Press, Accepted Manuscript.

Dräger, B., Jansen, A., Bruchmann, S., Förster, A.F., Pleger, B., Zwitserlood, P., et al. (2004). How does the brain accommodate to increased task difficulty in word finding?: A functional MRI study. Neuroimage, 23(3), 1152-1160.

Fernaeus, S.E., Ostberg, P., Almkvist, O. \& Wahlund, L.O. (2000). Differential temporal patterns in category and letter-based fluency: Implications for diagnosis and localization. Neurobiology of Aging, 21(Supplement 1), 159-159.

Fu, C.H. Y., Curtis, V.A., Williams, S.C.R., Brammer, M.J., Vythelingum, N., Morgan, K., et al. (2000). Overt verbal fluency examined with fMRI. Neuroimage, 11(5, Supplement 1), S338-S338.

Fu, C.H. Y., McIntosh, A.R., Kim, J., Chau, W., Bullmore, E.T., Williams, S.C.R., et al. (2006). Modulation of effective connectivity by cognitive demand in phonological verbal fluency. Neuroimage, 30(1), 266-271.

Gauthier, C.T., Duyme, M., Zanca, M. \& Capron, C. (2009). Sex and performance level effects on brain activation during a verbal fluency task: A functional magnetic resonance imaging study. Cortex, 45(2), 164-176.

Hair, A., Anderson, R.E., Tatham, E. \& Black, H. (Eds.). (1999). Análisis multivariante ( 5 ed.).

Hasan, K.M., Iftikhar, A., Kamali, A., Kramer, L.A., Ashtari, M., Cirino, P.T., et al. (2009). Development and aging of the healthy human brain uncinate fasciculus across the lifespan using diffusion tensor tractography. Brain Research, 1276, 67-76.

Henry, J.D. \& Crawford, J.R. (2004). A Meta-Analytic Review of Verbal Fluency Performance Following Focal Cortical Lesions. Neuropsychology, 18(2), 284-295.

Huettel, S.A., Misiurek, J., Jurkowski, A.J. \& McCarthy, G. (2004). Dynamic and strategic aspects of executive processing. Brain Research, 1000(1-2), 78-84.

Huizinga, M., Dolan, C.V. \& Van der Molen, M.W. (2006). Age-related change in executive function: Developmental trends and a latent variable analysis. Neuropsychologia, 44(11), 2017-2036.

Jefferies, E., Patterson, K. \& Ralph, M.A.L. (2008). Deficits of knowledge versus executive control in semantic cognition: Insights from cued naming. Neuropsychologia, 46(2), 649-658.

Johnson, M.H. (2003). Development of human brain functions. Biol Psychiatry, 54(12), 1312-1316.

Koren, R., Kofman, O. \& Berger, A. (2005). Analysis of word clustering in verbal fluency of school-aged children. Archives of Clinical Neuropsychology, 20(8), 1087-1104.

Lezak, M. (1995). Neuropsychological Assessment.

Marino, J. \& Alderete, A. (2010). Valores Normativos de Pruebas de Fluidez Verbal Categoriales, Fonológicas, Gramaticales y Combinadas y Análisis Comparativo de la Capacidad de Iniciación. Revista de Neuropsicología Neuropsiquiatría y Neurociencias, 10(1), 79-93.

Miyake, A., Friedman, N.P., Emerson, M.J., Witzki, A.H., Howerter, A. \& Wager, T.D. (2000). The Unity and Diversity of Executive Functions and Their Contributions to Complex "Frontal Lobe" Tasks: A Latent Variable Analysis. Cognitive Psychology, 41(1), 49-100. 
Noonan, K., Jefferies, E., Corbett, F. \& Lambon Ralph, M. (2009). Elucidating the Nature of Deregulated Semantic Cognition in Semantic Aphasia: Evidence for the Roles of Prefrontal and Temporoparietal Cortices. Journal of Cognitive Neuroscience, 22(7), 15971613.

Ostberg, P., Crinelli, R.M., Danielsson, R., Wahlund, L.O., Bogdanovic, N. \& Fernaeus, S.E. (2007). A Temporal Lobe Factor in Verb Fluency. Cortex, 43(5), 607-615.

Piatt, A.L., Fields, J.A., Paolo, A.M. \& Tröster, A.I. (1999). Action (verb naming) fluency as an executive function measure: convergent and divergent evidence of validity. Neuropsychologia, 37(13), 1499-1503.

Pulvermüller, F. (2002). A brain perspective on language mechanisms: from discrete neuronal ensembles to serial order. Progress in Neurobiology, 67(2), 85-111.

Schwartz, S. \& Baldo, J. (2001). Distinct patterns of word retrieval in right and left frontal lobe patients: a multidimensional perspective. Neuropsychologia, 39(11), 1209-1217.

Schwartz, S., Baldo, J., Graves, R.E. \& Brugger, P. (2003). Pervasive influence of semantics in letter and category fluency: A multidimensional approach. Brain and language, 87(3), 400-411.

Shtyrov, Y., Kujala, T. \& Pulvermüller, F. (2010). Interactions between Language and Attention Systems: Early Automatic Lexical Processing? Journal of Cognitive Neuroscience, 22(7), 1465-1478.

Tröster, A.I., Woods, S.P., Fields, J.A., Hanisch, C. \& Beatty, W.W. (2002). Declines in switching underlie verbal fluency changes after unilateral pallidal surgery in Parkinson's disease. Brain and Cognition, 50(2), 207-217.

Troyer, A.K., Moscovitch, M. \& Winocur, G. (1997). Clustering and Switching as Two Components of Verbal Fluency: Evidence From Younger and Older Healthy Adults. Neuropsychology, 11(1), 138146.

Troyer, A.K., Moscovitch, M., Winocur, G., Alexander, M.P. \& Stuss, D. (1998). Clustering and switching on verbal fluency: the effects of focal frontal and temporal lobe lesions. Neuropsychologia, 36(6), 499-504.

Vitali, P., Abutalebi, J., Tettamanti, M., Rowe, J., Scifo, P., Fazio, F., et al. (2005). Generating animal and tool names: An fMRI study of effective connectivity. Brain and language, 93(1), 32-45.

Voineskos, A.N., Rajji, T.K., Lobaugh, N.J., Miranda, D., Shenton, M. E., Kennedy, J.L., et al. (2010). Age-related decline in white matter tract integrity and cognitive performance: A DTI tractography and structural equation modeling study. Neurobiology of Aging, In Press, Corrected Proof. 